\title{
BMJ Open How do doctors and nurses manage delirium in intensive care units? A qualitative study using focus groups
}

\author{
Domingo Palacios-Ceña, ${ }^{1,2}$ José Miguel Cachón-Pérez, ${ }^{3}$ Rosa Martínez-Piedrola, ${ }^{1}$ \\ Javier Gueita-Rodriguez, ${ }^{1}$ Marta Perez-de-Heredia, ${ }^{1}$ \\ Cesar Fernández-de-las-Peñas ${ }^{1}$
}

To cite: Palacios-Ceña D, Cachón-Pérez JM, MartínezPiedrola $\mathrm{R}$, et al. How do doctors and nurses manage delirium in intensive care units? A qualitative study using focus groups. BMJ Open 2016;6:e009678. doi:10.1136/bmjopen-2015009678

- Prepublication history for this paper is available online. To view these files please visit the journal online (http://dx.doi.org/10.1136/ bmjopen-2015-009678).

Received 12 August 2015 Revised 21 September 2015 Accepted 13 October 2015

CrossMark

\begin{abstract}
${ }^{1}$ Physical Therapy, Occupational Therapy, Rehabilitation and Physical Medicine, Universidad Rey Juan Carlos,

Alcorcon/Madrid, Spain

${ }^{2}$ Grupo Excelencia

Investigadora URJC-Banco

Santander referencia N³0VCPIGI03: Investigación traslacional en el proceso de salud - enfermedad (ITPSE), Universidad Rey Juan Carlos, Alcorcon/Madrid, Spain). ${ }^{3}$ Department of Nursing, Universidad Europea de Madrid, Villaviciosa de Oon, Spain
\end{abstract}

Correspondence to Dr Domingo Palacios-Ceña; domingo.palacios@urjc.es

\section{ABSTRACT}

Objectives: The aim of this study was to explore the experiences of doctors and nurses caring for patients with delirium in the intensive care unit (ICU) and to describe the process of delirium management.

Setting: This study was performed in 5 ICUs located within 4 hospitals in Madrid (Spain).

Participants: Purposeful sampling was performed which included (1) doctors and nurses working in ICUs, (2) with >1 year experience in the ICU and (3) clinical experience with delirium. 38 professionals participated (19 doctors, 19 nurses), including 22 women and 16 men. The total mean age was 39 years. Design: A qualitative study using focus groups.

Methods: 7 focus groups were held to collect data: 3 nurse focus groups, 3 doctor focus groups and 1 mixed focus group. Each group comprised 6-10 participants. A semistructured questions guide was used. Thematic analysis methods were used to analyse the data.

Results: 3 themes were identified: (1) the professional perspective on delirium; (2) implementing pharmacological and non-pharmacological treatment for delirium and (3) work organisation in the ICU. The professionals regarded patients with delirium with uncertainty, and felt they were often underdiagnosed and poorly managed. Doctors displayed discrepancies regarding pharmacological prescriptions and decisionmaking. The choice of medication was determined by experience. Nurses felt that, for many doctors, delirium was not considered a matter of urgency in the ICU. Nurses encountered difficulties when applying verbal restraint, managing sleep disorders and providing early mobilisation. The lack of a delirium protocol generates conflicts regarding what type of care management to apply, especially during the night shift. A degree of group pressure exists which, in turn, influences the decision-making process and patient care.

Conclusions: Patients with delirium represent complex cases, requiring the implementation of specific protocols. These results serve to improve the process of care in patients with delirium.

\section{INTRODUCTION}

Delirium is a syndrome characterised by an alteration of consciousness and cognitive
Strengths and limitations of this study

- This study presents new data on the perception of delirium management among doctors and nurses working in the intensive care units (ICUs) of four hospitals in Madrid (Spain).

- Focus groups were used as a method for qualitative data collection as they enable the analysis of a range of values and norms in a group of individuals with a similar background and concerns.

- The group moderator was key in promoting participation within the focus groups in order to encourage group discussion and sharing of varying perspectives and management criteria by asking each group member questions individually and offering them different scenarios to comment on.

- None of the researchers were doctors (they were 2 nurses, 2 occupational therapists and 2 physical therapists), which may have influenced data analysis; however, researcher triangulation was performed, whereby the cases were analysed and presented among the researchers in team sessions.

- The findings of this study may help to improve the implementation of specific protocols for patients with delirium admitted to ICUs.

functions ${ }^{1}{ }^{2}$ and presenting a variable and rapid course lasting for hours or days. ${ }^{2}{ }^{3}$ Three types of delirium are described according to its clinical presentation: hyperactive, hypoactive or mixed. The hyperactive form is characterised by psychomotor agitation, hallucinations and considerable restlessness and agitation, whereas the hypoactive form, which is more difficult to identify, presents a lethargic clinical picture, in which the person remains disconnected from their surrounds. Finally, the mixed form fluctuates between both phases. ${ }^{14}$

Delirium affects between $38 \%$ and $87 \%$ of all patients admitted to surgical and medical intensive care units (ICUs), ${ }^{1}{ }^{4-6}$ rising up to 
$77 \%$ in burn units ${ }^{7}$ and affecting between $60 \%$ and $80 \%$ of patients with mechanical ventilation. ${ }^{7}$

The main risk factors in ICUs are: age $>65$ years, dementia, severity of the illness, ${ }^{8}$ temperature $>38^{\circ} \mathrm{C}$, infections, respiratory disease, hypotension, metabolic acidosis, ${ }^{8} 9$ hypoxia, ${ }^{10}$ sepsis, use of opioids, ${ }^{11}$ mechanical ventilation and physical restrictions. ${ }^{812}$

Delirium presents complications such as the accidental removal of medical devices (catheters), the prolongation of mechanical ventilation, increased hospitalisation time, ${ }^{13}{ }^{14}$ increased costs, ${ }^{1}{ }^{15}$ increased mortality and morbidity, ${ }^{1}{ }^{13} 15{ }^{16}$ cognitive decline, ${ }^{15}{ }^{17-19}$ and a decreased quality of life. ${ }^{1} 15$

The management of delirium is based on a combination of pharmacological and non-pharmacological treatments. ${ }^{1} 13 \quad 15$ 20 The most common medication includes antipsychotics (haloperidol), agonists of $\alpha 2$-adrenergic (dexmedetomidine, clonidina) and sedatives, ${ }^{15}$ 20-23 whereas the non-pharmacological treatment is based on: environmental control of the ICU; pain management; $;{ }^{25}$ avoidance of sleep deprivation; $;{ }^{26}$ continuous delirium assessment; ${ }^{5}$ early mobilisation; ${ }^{24} 27$ and/or early combinations of mobilisation, removal of sedation and mechanical ventilation and the application of respiratory physiotherapy. ${ }^{20} 2425$

How are these treatments applied? What is the perspective of ICU professionals regarding patients with delirium? What obstacles and facilitators are there for providing treatments? The aim of this study was to explore the experiences of doctors and nurses faced with patients with delirium in ICUs and to describe the management process when dealing with delirium.

\section{METHODS}

\section{Design}

A qualitative exploratory study was performed using focus groups (FGs). Qualitative methods enable the study of complex realities ${ }^{28}$ such as the meaning of illness and health. ${ }^{29}{ }^{30}$ They facilitate the study of medical collectives, the relation between patients and professionals, ${ }^{31}$ and provide information regarding how healthcare is organised, as well as the management of health services. ${ }^{32}$

A FG is a method of qualitative data collection, ${ }^{33-35}$ used to study unexplored situations or those that are difficult to access via other methods. As well as confirming the hypothesis, developing questionnaires and designing intervention programmes, ${ }^{34}$ FGs have been used in the ICU for the study of family participation during ICU rounds, ${ }^{36}$ the cultural competence of ICU professionals, ${ }^{37}$ the identification of barriers for enteral feeding in critically ill patients and the role of the nurse in the identification of delirium and the application of non-pharmacological treatment. ${ }^{38}$

\section{Research team}

Six researchers (4 men and 2 women) participated in this study, four of whom had experience in qualitative study designs (DP-C, JMC-P, RM-P and CF-d-l-P). All had $\mathrm{PhDs}$ in health sciences, were not involved in clinical activity, and had no prior relation with the participants. Prior to the study, the positioning of the researchers was established regarding the theoretical framework, the researchers' beliefs, their prior experience and their motivation for the research. ${ }^{39}{ }^{40}$ Researchers based their approach on a constructivist paradigm. This paradigm was based on the assumption that human beings construct their own social reality, and that knowledge is built through increasingly nuanced reconstructions of individual or group experiences. ${ }^{33}$ The main belief was that professionals in the ICU work in a coordinated manner, according to a specific protocol, and that good doctornurse communication exists in individuals who present with delirium. Finally, only three researchers had ICU experience (DP-C, JMC-P and JG-R).

\section{Participants}

Purposeful sampling was performed by selecting cases or units (individuals, groups of individuals, institutions) based on their capacity to provide relevant information in response to the research questions. ${ }^{41}$ Participants with relevant information were recruited, that is, doctors and nurses from the ICU. Purposeful sampling methods are recommended for studies using FGs. ${ }^{35} 4243$

The inclusion criteria consisted of: (1) doctors and nurses who worked in the ICU during the study, (2) professional experience in the ICU $>1$ year and (3) with experience in the management of patients with delirium.

The first contact was established with the medical director and nursing supervisor who placed the researchers in touch with the potential participants. During a second face-to-face meeting, the study was explained to the professionals. Finally, 2 weeks later, a third meeting took place during which those who wished to participate were confirmed and informed consent was obtained.

Thirty-eight professionals participated in the study, of whom 19 were doctors and 19 were nurses. These included 22 women and 16 men. The mean age of the participants was 39.39 years; the mean age of the nurses was 34.42 years, whereas that of doctors was 42.36 years. The mean experience of working in ICUs was 12.15 years; the mean working experience of nurses was 9.89 years, whereas that of doctors was 14.42 years. There were no dropouts (table 1).

\section{Setting}

The study was performed with participants from five ICUs working at four hospitals in Madrid (Spain). The ICUs had 12-20 beds but no individual rooms. The nurse/ patient ratio was 1:3, and the doctor/patient ratio was 1:1. The ICU types were multipurpose (respiratory-general surgery), surgical, trauma and coronary. All the ICUs were 'closed', with limited access, limited visiting hours and a limited number of family member visits allowed. ${ }^{44}$ Data collection was performed both at the Universidad Rey Juan Carlos and at the workplace of the participants. 
Table 1 Characteristics of the study participants and focus groups (FGs)

\begin{tabular}{|c|c|c|c|c|c|}
\hline FG & $\begin{array}{l}\text { Duration } \\
\text { (minutes) }\end{array}$ & Participants & Age, mean (SD) & Sex & $\begin{array}{l}\text { Experience in } \\
\text { intensive care, } \\
\text { mean (SD) }\end{array}$ \\
\hline FG1 & 105 & $\mathrm{n}=7$ Nurses & $34.85( \pm 6.09)$ & $\begin{array}{l}4 \text { Female } \\
3 \text { Male }\end{array}$ & $8.57( \pm 4.72)$ \\
\hline FG2 & 95 & $\mathrm{n}=6$ Nurses & $32.16( \pm 4.35)$ & $\begin{array}{l}4 \text { Female } \\
2 \text { Male }\end{array}$ & $9.5( \pm 4.67)$ \\
\hline FG3 & 110 & $\mathrm{n}=6$ Nurses & $36.16( \pm 4.11)$ & $\begin{array}{l}3 \text { Female } \\
3 \text { Male }\end{array}$ & $11.83( \pm 4.95)$ \\
\hline FG4 & 100 & $\mathrm{n}=6$ Doctors & $44.66( \pm 4.50)$ & $\begin{array}{l}4 \text { Female } \\
2 \text { Male }\end{array}$ & $17.66( \pm 6.02)$ \\
\hline FG5 & 80 & $\mathrm{n}=6$ Doctors & $42.83( \pm 6.75)$ & $\begin{array}{l}3 \text { Female } \\
3 \text { Male }\end{array}$ & $14( \pm 8.31)$ \\
\hline FG6 & 124 & $\mathrm{n}=7$ Doctors & $40( \pm 6.28)$ & $\begin{array}{l}4 \text { Female } \\
3 \text { Male }\end{array}$ & $12( \pm 6.21)$ \\
\hline FG7 & 118 & $\begin{array}{l}\mathrm{n}=10 \text { ( } 5 \text { Nurses and } \\
5 \text { doctors) }\end{array}$ & $37.5( \pm 5.46)$ & $\begin{array}{l}5 \text { Female } \\
5 \text { Male }\end{array}$ & $11.8( \pm 4.23)$ \\
\hline $\begin{array}{l}\text { Total } \\
\text { number } \\
\text { of FGs }\end{array}$ & $\begin{array}{l}\text { Duration } \\
\text { (minutes), } \\
\text { mean (SD) }\end{array}$ & $\begin{array}{l}\text { Total } \\
\text { participants }\end{array}$ & $\begin{array}{l}\text { Total mean age, } \\
\text { mean (SD) }\end{array}$ & $\begin{array}{l}\text { Total distribution } \\
\text { according to sex }\end{array}$ & $\begin{array}{l}\text { Experience in } \\
\text { intensive care } \\
\text { Total years, mean (SD) }\end{array}$ \\
\hline $\mathrm{N}=7$ & $10457( \pm 14.74)$ & $\mathrm{N}=38$ & $38.39( \pm 6.74)$ & $\begin{array}{l}22 \text { Female } \\
16 \text { Male }\end{array}$ & $12.15( \pm 6.28)$ \\
\hline
\end{tabular}

\section{Data collection}

FGs were held in order to examine different perspectives within the same group, acquire understanding of the problems faced by the group and aid the identification of values and norms. ${ }^{33} 35$

The FGs were constructed on the basis of the criteria of homogeneity, comprising participants working in ICUs (doctors and nurses). ${ }^{34}{ }^{45}$ In order to assign participants to each FG, these were first divided by profession (doctors and nurses), and thereafter the professionals were randomly distributed, maintaining the same proportion of participants in order to ensure uniform numbers in all the FGs. Seven FGs were formed, without exceeding the limit of 10 , as a higher number would not provide further relevant information. ${ }^{46}$ In total, there were three nurse FGs, three doctor FGs, and a mixed group including both doctors and nurses. Initially, the FGs were conducted separately for the doctors and the nurses in order to examine their independent perspectives. Thereafter, a mixed FG took place to analyse how professional groups described their perspectives and the differences between them, as well as to examine how a common framework was established that integrated both perspectives. Each FG comprised between 6 and 10 participants, as group sizes below 6 can make it difficult to sustain a meaningful discussion and a group of $>10$ may prove difficult to manage. ${ }^{46}$ The mixed FG was randomly formed from the total of participants of the previous FG, with a proportional number of doctors and nurses (see table 1). Randomisation was performed in order to avoid a selection bias within the nurse and doctor groups. Proportional numbers of doctors and nurses were established in order to avoid having more members in any of these professional groups, as well as to avoid imposing a single perspective.

\section{FG procedure}

The FGs followed a uniform structure, ${ }^{43} 47$ and were conducted by a moderator and an observer. The moderator posed questions to which each participant responded, respecting their turn to speak. Thereafter, the moderator posed further questions, based on the issues that were brought up in the discussion, in order to explore further or clarify aspects, either individually or to the group as a whole. ${ }^{47}$ The observer supported the moderator, identifying key points and taking field notes (table 2).

FGs were conducted in Spanish. Prior to data collection, all participants had participated in a refresher course on delirium organised within their respective ICU units. In these sessions, they all used the same definition for delirium based on the Diagnostic and Statistical Manual of Mental Disorders, Fourth Edition, Text Revision (DSM-IV-TR). ${ }^{48}$

A questions guide was used, ${ }^{43} 47$ which was centred enough in order to gather information on the area of study, but open enough to stimulate discussion and interaction among the participants. ${ }^{34}$

All the FGs were audio recorded. Permission for recording the FGs was sought prior to performing the recordings. The total duration of the recordings was $732 \mathrm{~min}$, with a mean duration of $104.57 \mathrm{~min}$.

Data collection was pursued until the researcher achieved information redundancy, at which point no new information emerged from data analysis (in our study, this occurred in FG7). ${ }^{33}$ Participants were 
Table 2 Structure and phases of focus groups

\begin{tabular}{|c|c|c|}
\hline Phase & Contents & $\begin{array}{l}\text { Time } \\
\text { (minutes) }\end{array}$ \\
\hline Moderator welcome & Welcome. Explanation of study aims, process of the session and rules & $5-10$ \\
\hline Opening question & $\begin{array}{l}\text { Participants were asked about their experience with patients with delirium: Could you tell } \\
\text { me your experience with patients who have had delirium? }\end{array}$ & $10-20$ \\
\hline $\begin{array}{l}\text { Introductory and } \\
\text { transition questions }\end{array}$ & $\begin{array}{l}\text { The question was centred on aspects of delirium prevention and management } \\
\text { How was delirium identified? } \\
\text { What prevention activities were you performing? What activities (treatment, care) were } \\
\text { performed for the management of delirium? }\end{array}$ & 10-30 \\
\hline Key questions & $\begin{array}{l}\text { Questions were posed once more on the basis of prior responses of participants in order to } \\
\text { go into greater depth regarding areas such as: doctor-nurse relations: Do you think the } \\
\text { relations among different professionals may influence the prevention and management of } \\
\text { delirium? } \\
\text { Protocol development in the ICU: What is your experience with protocols in the ICU? How } \\
\text { are these developed? Patient safety: What is your experience regarding delirium and } \\
\text { patient safety? How does your intervention contribute towards maintaining patient safety? }\end{array}$ & $20-40$ \\
\hline Closing remarks & $\begin{array}{l}\text { The moderator performed a brief summary of the contents covered. } \\
\text { The participants confirmed whether the summary was correct and were given the } \\
\text { opportunity to add to the same }\end{array}$ & $10-15$ \\
\hline
\end{tabular}

provided with a copy of the transcripts from the FGs in order to grant them the opportunity to provide further comments or corrections.

\section{Data analysis}

Three researchers with experience in qualitative studies (DP-C, JMC-P and RM-P) performed the analysis of the FG data. First, an analysis of each FG was performed. Afterwards, the results of the initial analysis were subsequently merged in joint sessions, during which collection and analysis procedures were discussed. In the case of different opinions, theme identification was decided by consensus.

Data collection included a full literal transcription of the FGs and the researcher field notes. ${ }^{33} \mathrm{~A}$ thematic analysis $^{49}$ approach was used on the data, involving an initial descriptive analysis of the transcribed texts (words, sentences and metaphors directly from the text). The data collected were then reduced to meaning units in order to identify emerging topics. Subsequently, meaning units were clustered into common meaning groups in order to define the main topics. The final outcome was the identification of themes emerging from the data. ${ }^{50}$ Participants were provided with the analysis from their FG in order to gather their feedback. ${ }^{40}$ No qualitative software was used on the data.

\section{Quality}

Our report follows the recommendations established by the consolidated criteria for reporting qualitative research. ${ }^{40}$ The data verification method used consisted of: (1) cross-triangulation by the researcher, involving planning sessions whereby the cases analysed by each team member were presented with the objective of reaching consensus (the purpose of triangulation was for completeness of data, as triangulation enables a more contextual portrayal of phenomena, which may enrich understanding) $;^{51}$ (2) auditing of the materials obtained from two randomly selected FGs (FG3 and FG4) by an external independent researcher (the external researcher reviewed the quality of the process of data collection and analysis) and (3) post focus-group analysis (transcripts returned) and verification by participants post analysis (participant checking).

Informed consent was obtained from participants prior to their participation in the study.

\section{RESULTS}

The study's duration was from 1 September 2012 to 20 April 2014. Three themes were identified: (1) the professional perspective on delirium; (2) implementing pharmacological and non-pharmacological treatment of delirium, including two subthemes: pharmacological treatment and non-pharmacological treatment and (3) work organisation in the ICU.

Each of the themes is accompanied by extracts from the accounts of the participants, in order to improve the credibility of the results (table 3 ). ${ }^{40}$

\section{Theme: the professional perspective on delirium}

Most professionals faced delirium with a great sense of doubt. Doctors described the complexity of the physiopathology, which hampers the management of the same. Patients with delirium generate unrest and stress. They are unable to control themselves and therefore involve constant dedication on behalf of the staff. All professionals described the feeling of not having enough therapeutic tools to manage these cases. Most professionals felt that these patients are often underdiagnosed 
Table 3 Representative quotations from study participants

The professional perspective on delirium
Grey areas in
delirium management

"I feel uncomfortable by the situation as I do not control it, I can't

understand it, whereas I do understand things like the physiopathology of shock. Delirium is something that goes beyond our intellectual management $(. .$.$) the other pathologies we control by managing the$ causes, but delirium is different..." (FG4l6, 47 years, female)

Need for constant attention

Underdiagnosis

Stereotypes regarding patients with delirium

Responsibility and nursing

Different assessment criteria

Hypoactive delirium

Pharmacological
treatment

Implementing pharmacological and non-pharmacological delirium treatment

"We have to wait for the medication to have an effect, but, in the meantime, it is really disheartening, sometimes the medication doesn't even work, and at other times you have to wait for the patient to be assessed by the doctor. You don't know what to do with them, which is stressful because you have to attend to other patients and meanwhile they remove their catheters..." (FG1E2, 38 years, female) "In trauma ICUs, a patient with delirium is never diagnosed. It is a manifestation of the brain lesion and that's all." (FG2E8, 27 years, female)

"Typical scenario: an older patient, who is admitted, occupies all your attention because you expect them to give you a bad night, get distressed, remove catheters, etc. But, in the end, it is the adult, sedated, under analgesia, and with electrolytical alterations who gets agitated. You try to focus on age, when there are many other warning signs."(FG7I3, 40 years, female)

"... in the end, the patient with delirium is a responsibility for nurses, the doctor either doesn't like it or doesn't want to see the patient, and says that there is nothing they can give the person, so you can't leave the bedside, and you have no means to manage these patients..." (FG3E17, 37 years, male)

"When the intensive care doctors go in the morning to assess a disoriented patient who has been agitated all night, the assessment is almost always more positive, they see the patient more oriented than we do." (FG1E4, 33 years, male)

"Patients with hypoactive delirium are the most abandoned patients, they don't demand anything, they don't move and don't do anything, they are the ideal patient for intensive care..." (FG3E16, 39 years, female)
There is no medication of choice

\section{Discrepancies} between the medical prescription and the dosage

The drug choice is determined by the experience of use

Delirium is not a life-threatening emergency

Different treatment in each shift
"You don't have a specific drug for treating delirium, and the few that exist have many side effects, despite administrating the recommended dosage. I have had to intubate a patient due to the effects of the treatment."(FG6I18, 31 years, female)

"I'm not sure of how to dose haloperidol, sometimes the patient is asleep all morning, but agitated all night, you don't know how to get it right."(FG5I7, 54 years, male)

"You just don't trust it, and you use what you trust the most. You have to be able to quickly and effectively control the patient, you can't wait to see whether a new drug will work because the commercial delegate tells you so."(FG5I10, 45 years, male)

"You spend your whole shift calling the doctor, it's as if the patient were your sole responsibility and there is no way they will prescribe you anything....when they do, they prescribe a negligible dose in relation to the patient's weight...and if it is night time, sometimes you have to wait, they do not consider it to be an emergency."(FG3E18, 29 years, male)

"Sometimes, you have a very sleepy patient or you have people throwing themselves out of the bed. Also, you know that in each shift, what one person prescribes, another will remove. Therefore you have patients who, on the same day may have tried 3 or 4 different drugs for delirium."(FG2E10, 39 years, female) 
Table 3 Continued

Nurses' demands

Non-pharmacological treatment

Verbal restraint: distrust

Verbal restraint: conflicts with its application

Mechanical restraint: indiscriminant use and most used remedy

Mechanical restraint: medical orders

Sleep management

Healthy vs hostile environment

Early mobilisation

Theme: work organisation in the ICU

Absence of protocol and conflicts among nurses

Group pressure
"In the end, the nurses request a solution you they don't have, you don't know what to do, sometimes I have had to sedate a patient. During some shifts, you avoid the nursing station because you don't know what to tell them, there are no fast solutions, sometimes you have to be patient and wait, stay by the patient's side." (FG4I4, 43 years, male)

"At first, you try to explain, but soon you are left without any arguments and with no patience (...) I don't know if any of you have had any success, I don't know what you tell the patient, in what sort of tone, and when is the best time for doing so, but never in the 14 years I have worked in the ICU have I been successful, and I don't believe it works." (FG1E3, 39 years, female)

"It seems like you are "the odd one out", just because you spend some time trying to get to know what is wrong with the disoriented patient, or you lose time trying to orient them, knowing these are people who are bothering the other patients." (FG2E8, 27 years, female)

"Everyone got restraints. Nobody even considered that medical prescriptions were necessary." (FG4I3, 52 years, male) "In the end, when you don't know what to say to the patient, you end up using restraints, it is the last fix before pharmacological treatment, sometimes you use them while awaiting medical orders." (FG3E14, 41 years, male)

"As a nurse, you can't use restraints unless they are prescribed, but the doctor doesn't write this down either. In the end, it's the nurse who decides." (FG2E11, 28 years, male)

"Sleeping seems to be an impossible feat, the patient wakes up due to the noise of the machinery, noises made by other patients, or the high tone of our conversations" (FG2E9, 34 years, female)

"It is complicated trying to convince colleagues to keep their voices down during the night, sometimes the laughter is impossible to contain."(FG2E12, 35 years, male)

"If you try and put yourself in the place of the patient, all you feel is fear, you are surrounded by people who are strangers and everything beeps, it is impossible to rest."(FG7I5, 43 years, male)

"It seems simple, but to lift an intubated patient, with all the pumps, the monitor, all the cables, drainages, catheters and so on is not easy, nobody likes to do it, sometimes it is more risky to mobilise the patient than to wait for delirium to appear and then manage it..."(FG3E15, 35 years, male) "Is it really necessary to wake the patient in order to take his temperature at three in the morning, or wake him up to administer sleeping medication?" (FG2E9, 34 years, female)

"... at night time, you are bothering patients every hour, either to take their temperature, or due to the urine, or for the medication, or for a scale (...) the patient is easily woken, gets scared, and ends up getting disoriented. This way of working does not favor the patient's rest or wellbeing."(FG7E5, 38 years, female)

"There is nothing established, there are no protocols on favoring sleep, at times you have to confront your colleagues, and in the end, the easiest thing is to agree on which care measures are going to be applied during that shift." (FG3E15, 35 years, female)

"There are no protocols, you have to be there 24 hours a day, working at full capacity, we do nothing to modify our acts, perhaps we are actually part of the problem, we are lacking protocols in order for all of us to work along the same lines." (FG6I16, 52 years, male)

"You know that the patient doesn't need his vital signs taken every hour, once a night would be enough, but if you want to be left alone and not be criticized you keep on noting down their vital signs every hour, although you waken the patient and know that he should really sleep."(FG7E3, 27 years, female)

FG, focus group; ICU, intensive care unit. 
and undertreated, as delirium is considered to be a clinical symptom of other pathologies and not a problem in itself.

Another widespread belief was that delirium only appears in the elderly. Ironically, this caused professionals to be more attentive to these patients, although other adults may actually be more at risk. Nurses perceived patients with delirium as problematic, and felt that the burden of responsibility for these patients is on the health professional. Also, nurses shared their experiences of doctors tending to perform more positive neurological assessments, compared with their own nursing assessments. Nurses believed that their assessments were more accurate as these are performed continuously, compared with the more sporadic medical assessments. For nurses, hypoactive delirium was regarded as the most dangerous type of delirium as the patient is undemanding and thus considered to be 'good' due to the fact that they do not require excessive care.

\section{Theme: implementing pharmacological and non-pharmacological delirium treatment}

This theme describes interventions targeted at improving delirium via the use of pharmacological and nonpharmacological treatment.

\section{Subtheme: pharmacological treatment}

Doctors described not having a drug of reference for the treatment of delirium. The most commonly used medication included haloperidol and dexmedetomidine, although these were reported to have varying degrees of success. Thus, different opinions were gathered regarding dosage and guidelines for the same. Many doctors acknowledged that the choice of a drug is determined more by the degree of experience and confidence in managing it, rather than by its efficacy.

For most nurses, doctors did not consider patients with delirium to be 'urgent cases', often prescribing pharmacological doses below the therapeutic range. This was a problem that worsened during the night shift. Also, they described how doctors used a wide range of pharmacological drugs according to their criteria and experience, without agreeing on a clear and continuous guideline throughout the different shifts. This resulted in some patients receiving different treatments according to the doctor on shift.

Doctors felt that nurses were demanding a solution that they did not have as they were unaware of the physiopathological cause of delirium and were lacking a treatment of choice. They acknowledged that on occasion, these issues made them delay their communications with the nurses.

\section{Subtheme: non-pharmacological treatment}

This includes measures such as verbal restraint, mechanical restraint and sleep management, providing a healthy environment and early mobilisation.
Verbal restraint

Most professionals agreed that verbal restraint is the first intervention measure used, but they distrusted its efficacy in achieving patient reorientation and decreasing the clinical symptoms. Also, the use of verbal restraint was sometimes a source of tension among nurses, as it was often met with scepticism by colleagues working on the night shift.

\section{Mechanical restraint}

Most professionals reported the indiscriminate use of restraints. Mechanical restraints were the most widely used remedy when verbal restraint failed. Some nurses reported that mechanical restraints were a measure they used while 'on hold' for more precise medical orders. All nurses felt that the use of restraint should be clearly prescribed by the doctor. Generally, owing to the lack of orders, nurses were forced to make these decisions on the go.

\section{Management of sleep}

All professionals perceived that patients admitted to the ICU had great difficulty in sleeping and obtaining the necessary rest.

\section{Healthy environment}

All professionals gave special importance to the control of noise and agreed that it was a source of risk for the development of delirium. The sources of noise reported included night-time conversations between professionals, the sound of alarms ringing and beeping and the environmental noise within the ICU (movements of staff). Most participants described the environment of the ICU as hostile, acknowledging that it was not the appropriate environment for patients suffering from delirium.

\section{Early mobilisation}

Early mobilisation was perceived as complicated to perform and fraught with difficulties, due to the lack of personnel, equipment (ie, hoists) and organisation (during which shift it should take place).

\section{Theme: work organisation in the ICU}

Aspects involving work organisation (timetables, techniques, personnel routines) were seen to influence the appearance of delirium. Nurses associated the appearance of delirium with night-time care. Most participants questioned the need for constantly waking the patient in order to collect data as this could undermine the patient's rest. They believed that the timing of patient care could iatrogenically provoke delirium.

The ICUs in which the nurses included in this study worked did not have a specific protocol for the management of delirium. As a result, diverse criteria existed, leading to conflicts on deciding which care pathway was the most suitable. In these cases, it was necessary to reach an agreement with colleagues. Professionals demanded the need for implementing specific protocols 
for delirium management. This would aid the decisionmaking process, the distribution of work, the control over patients, as well as decrease conflicts.

An element of group pressure was identified within the organisation of nursing work, which influenced the decision-making process and patient care. This pressure was influenced by: seniority, duty and group consensus. Regarding seniority, the approval of more experienced nurses was deemed necessary in order to perform certain care measures. A sense of duty was acknowledged as nurses felt they could not stop performing certain care measures because these were seen as part of their duty 'as nurses' (timetable control, postural changes), despite the fact that these procedures could cause patients discomfort, and because failure to do so could lead to criticism by the nurses in the ensuing shift. Finally, a 'non-written rule' was described in which the implementation of new protocols or care procedures had to be approved by the remaining nurses in the ICU. Things could not be performed differently or against the group's opinion. Some nurses described a feeling of fear associated with performing changes to an already established work routine or any other innovation.

\section{DISCUSSION}

The main strength of this study is the description of how delirium is managed by doctors and nurses in ICUs, presenting first-time accounts that can influence patient care. To the best of the authors' knowledge, this is one of the few studies that describes how pharmacological and non-pharmacological treatments are applied in patients with delirium in ICUs. ${ }^{38} 52$ In Spain, Cachón-Pérez et $a l^{38}$ described how, according to the professional perspective, the physical structure of ICUs, the degree of family involvement and the training of nurses all help to decrease the delirium. The aforementioned study coincides with our results in that, on the one hand, it is necessary to control and favour patient sleep, although the dynamics within the ICU make this difficult. On the other hand, there is a degree of group pressure among nurses which conditions the application of patient care. This type of pressure, in the form of the imposition of criteria or criticisms regarding the quality of the work performed, has not been found in other studies. On an international level, Jung et $a \bar{l}^{2}$ have described how the nurses in ICUs failed to use any type of specific protocol for the detection of delirium, which coincides with our findings, and how the use of qualitative research methods enabled the identification of training requirements among ICU nurses. Another strength of this study is that we used a similar method (qualitative methods with FGs) as previous studies researching delirium in the ICU. ${ }^{38}$ Further, we were able to use a proportionate number of participants in all the FGs.

This study has some limitations. The first is that the results cannot be extrapolated; however, they can be applied to contexts with similar characteristics. The second is that none of the researchers was a doctor, and therefore this could influence the results. Third, the dynamics and the data collected from the mixed group may have altered the results. This limitation was controlled, as previously we performed doctor and nurse FGs separately in which they described their perspectives. The mixed FG provided further information, which confirmed some of the results obtained separately in the previous FGs. For example, both professional groups coincided in the need for the nurse to have a fast and effective treatment for delirium. Finally, within the FGs, the participants can tend to unify their criteria, and avoid comparison and dialogue, which may produce uniform results. ${ }^{34}$ Of note is that one of the weaknesses of our qualitative results is that it may be difficult to apply these findings to other ICUs with different cultural and geographic settings. This has been controlled via the exhaustive description of the context and methods, thus enabling other researchers to apply the same in other contexts (ie, ensuring transferability). ${ }^{33}$

Currently, the Pan American and Iberian Federation of Societies of Intensive and Critical Care Medicine, ${ }^{53}$ the American College of Critical Care Medicine, ${ }^{54}$ and the National Institute for Health and Care Excellence $^{55} 56$ have determined that good evidence exists for the following practices concerning delirium management in the ICU: (1) routine monitorisation of ICU patients for delirium (the Confusion Assessment Method for the ICU (CAM-ICU) is one of the most valid and reliable instruments to assess delirium in the ICU), (2) early mobilisation to reduce the incidence and duration of delirium and to improve functional outcomes, (3) sleep promotion in ICU patients by controlling light and noise, clustering patient care activities, and decreasing stimuli at night and (4) pharmacological treatment of delirium: use of haloperidol as a first-line pharmacological agent, use of olanzapine as an alternative for the short-term treatment of delirium, when sedation is required in delirious patients, use of dexmedetomidine rather than benzodiazepine, the use of antipsychotics must be avoided in those patients with baseline QT prolongation, and avoidance of rivastigmine in ICU patients.

Our results show how the staff's own 'beliefs' can determine their perspectives on delirium (for example, the belief that delirium is more common in the elderly). Furthermore, the staff's knowledge and prior education surrounding delirium can condition their understanding regarding the management of delirium in the ICU. ${ }^{57}$ Elliot $^{57}$ showed that nurses receive insufficient training in ICUs regarding delirium, and consequently they have a poor level of knowledge regarding the associated complications and risk factors. Also, there is a tendency to not use a specific assessment tool on a daily basis for the detection of delirium.

The ICUs analysed in our study had no specific protocols for the diagnosis and treatment of delirium, and doctors and nurses referred difficulties caring for these 
patients. Similarly, Fan $e t a{ }^{58}$ also described the underdiagnosis and undertreatment of delirium cases in ICUs and how this can delay the care and cause inappropriate patient control. The application of specific protocols is helpful for unifying criteria, facilitating the working relation between different professionals managing delirium in ICUs. ${ }^{59}$ Participants in our study described the use of a specific instrument for delirium detection: the CAM-ICU. ${ }^{54}$ However, they use it in a non-structured way for the clinical assessment of delirium detection. This can lead to problems as this instrument focuses on signs such as hallucinations and agitation which are associated with hyperactive delirium, and thus it fails to detect other types of delirium, ${ }^{57}$ such as hypoactive delirium, which has been shown to be associated with prolonged delirium. ${ }^{56}$

Verbal restraint and patient reorientation are some of the first actions within the non-pharmacological strategies most commonly used by ICU professionals. ${ }^{11}$ However, despite the simplicity of these measures, their implementation presents considerable difficulties due to a lack of training and a lack of time on behalf of the staff. $^{43}$

The patient's own safety and the need for patients to avoid withdrawing equipment are the main justifications for the use of physical restraint in ICUs. ${ }^{24} 60$ Prior studies have shown how delirium is associated with the withdrawal of equipment and lesions ${ }^{60} 61$ and how the use of physical restraint is not justified as a first option. ${ }^{24}$ The nurses in our study knew that they must not apply physical restraint without a medical prescription; however, when they did do so, they justified it on the grounds of the patient's own safety. Safety is a recurrent motive for the use of restraints in patients with delirium. ${ }^{38}$

Previous studies have described how sleep deprivation can trigger episodes of delirium in ICU patients. ${ }^{26} 55$ Admittance to individual rooms, ${ }^{62}$ and the application of protocols targeted towards eliminating sleep disruptors are effective interventions for avoiding delirium. ${ }^{55}$ The role of nursing staff is fundamental, as some of the activities (changing bed linen, manipulation of venous catheters, the administration of medications, taking vital signs) are great sleep disruptors. ${ }^{63}$

The organisation of nursing work in ICUs and the application of care (maintenance of venous catheters, care of catheters and drainages and postural changes) can influence the appearance of delirium. ${ }^{64} 65$ The number of interventions should be minimised in order to favour patient participation and early mobilisation. ${ }^{64-66}$ This is a matter of particular importance during the night shifts in order to aid rest. Another factor with regard to organisation is the nurse/patient ratio. Reade and Finfer ${ }^{66}$ have described how, according to the number of beds in the ICU and the levels of care, the nurse/patient ratio can vary between $1: 1$ and 1:3. In the presence of patients with delirium, this ratio should decrease, assigning a single nurse for the care of each patient. ${ }^{38}$
It is noteworthy that the participants in this study described the ICU as a hostile environment, due to factors associated with their own activity such as the noise made by the staff, the night-time activity and the conversations among them. Despite recognising the influence of their presence as a source of discomfort, the participants were more focused on eliminating other factors, although the guidelines ${ }^{53-55}$ also point to their direct role as a risk factor for delirium.

Within a group or collective, there is an assignment of functions for each member, and a recognition of the social role occupied within the group. ${ }^{38}$ Linton and Farrell ${ }^{67}$ have described how the presence of a nursing hierarchy of care is related to the years of experience; that is, approximately 5 years are necessary in order to adopt the role of a leader. One of the functions of a leader is decision-making during situations of stress or difficulty. This has a positive influence on the work atmosphere in ICUs. However, according to our results, experience or seniority may be negatively perceived because it limits or conditions the application of new treatment approaches.

Our findings demonstrate that, during the process of delirium management, there are certain factors, such as the relation among professionals, their beliefs and their way of working, that may influence the application of treatments that have demonstrated to be effective. ${ }^{15} 2468$

These results may be applied: (1) in the clinic, for the development of specific protocols for the management of delirium in ICUs and (2) within ICU management, in order to perform changes in the organisation that support continuous care for patients with delirium throughout different nursing shifts, and for the development of interdisciplinary teams among doctors and nurses who evaluate delirium management in ICUs.

On the basis of our results, it would be necessary to establish the following changes in clinical practice: (1) increasing the education of ICU professionals, increasing knowledge on delirium ${ }^{57}$ and the use of recommended tools for delirium assessment; ${ }^{53-55}$ controlling for risk factors that are directly related to staff activity and (3) developing protocols that integrate the different perspectives of doctors and nurses, together with the clinical recommendations based on the evidence of delirium management in ICUs. ${ }^{53-55}$ In order to integrate the perspective of both professional groups and elaborate a protocol adapted to the specific context (type of ICU, culture and values), it is necessary to involve both in the process of protocol development. For this purpose, further research should be performed on the basis of participatory action research..$^{33}$ This type of design allows participants to go through a common process, sharing their perspectives, negotiating meanings and collaborating together in the development of programmes and projects for resolving common problems. ${ }^{69}$ In these types of designs, the participants are the fundamental basis of the protocol or elaborated project. 
On the other hand, one of the most commonly used strategies for delirium management and prevention, outside the context of the ICU, is the adoption of multidisciplinary care strategies. ${ }^{56}$ In the ICU, multidisciplinary care can be applied and strengthened, with the benefit of improving the coordination and communication among doctors and nurses.

\section{CONCLUSIONS}

Our findings highlight how professionals perceive individuals with delirium. Doctors have difficulties selecting the appropriate drug, and for some patients, the dosage of the same is seen to vary across different shifts. Nurses believe that for the doctor, delirium is not a matter of urgency, and therefore the attention is often delayed. On the other hand, nurses have difficulty in applying verbal restraint, sleep management and early mobilisation, and there is a tendency towards the use of physical restraint while awaiting medical recommendations. The absence of a delirium protocol generates conflicts regarding which path of care to apply, especially during the night shift. Also, a degree of group pressure was found among nurses, which directly influences patient care.

The complexity of delirium itself, together with the associated therapeutic variety, and the presence of difficulties in the application of care, can lead to inappropriate patient management. Individuals with delirium are complex patients requiring specific protocols that describe the acts to be performed by the different professionals who care for them. Also, the management of patients with delirium in the ICU must be based on teamwork between the doctor and nurse, featuring fluid, rapid and constant communication.

These findings may be applied to help identify the needs of the ICU staff caring for patients with delirium and for the elaboration of specific protocols that consider the professional's perspective. These results may help to understand how doctors and nurses apply decision-making processes regarding delirium management. This study contributes to the evidence base suggesting that delirium in the ICU presents obstacles to management despite current existing guidelines.

Acknowledgements The authors thank the nurses and doctors who agreed to participate in this study. They also thank Juan Francisco Velarde García for his contribution to the study.

Contributors DP-C, JMC-P and CF-d-I-P contributed to the conception and design, analysis, drafting of the work, gave final approval, and agreed for all aspects of the work. RM-P, JG-R and MP-d-H contributed to data collection, analysis, drafting of the work, gave final approval, and agreed for all aspects of the work.

Funding This research has received no specific grant from any funding agency in the public, commercial or not-for-profit sectors.

Competing interests None declared.

Patient consent Obtained.

Ethics approval This study was approved by the local ethical research committee of the Universidad Rey Juan Carlos, Madrid, Spain.
Provenance and peer review Not commissioned; externally peer reviewed.

Data sharing statement Additional data can be accessed via the Dryad data repository at http://datadryad.org/ with the doi:10.5061/dryad.5rq76.

Open Access This is an Open Access article distributed in accordance with the Creative Commons Attribution Non Commercial (CC BY-NC 4.0) license, which permits others to distribute, remix, adapt, build upon this work noncommercially, and license their derivative works on different terms, provided the original work is properly cited and the use is non-commercial. See: http:// creativecommons.org/licenses/by-nc/4.0/

\section{REFERENCES}

1. Jackson $P$, Khan A. Delirium in critically ill patients. Crit Care Clin 2015;31:589-603.

2. ÓMahoney R, Murthy L, Akunne A, et al. Synopsis of the National Institute for Health and Clinical Excellence guidelines for prevention of delirium. Ann Intern Med 2011;154:746-51.

3. Caplan JP, Rabinowitz T. An approach to the patient with cognitive impairment: delirium and dementia. Med Clin North Am 2010;94:1103-16.

4. Wong CL, Holroyd-Leduc J, Simel DL, et al. Does this patient have delirium?: value of bedside instruments. JAMA 2010;304:779-86.

5. Guenther U, Weykam J, Andorfer U, et al. Implications of objective vs subjective delirium assessment in surgical intensive care patients. Am J Crit Care 2012;21:e12-20.

6. Vasilevskis EE, Han JH, Hughes CG, et al. Epidemiology and risk factors for delirium across hospital settings. Best Pract Res Clin Anaesthesiol 2012;26:277-87.

7. Agarwal V, O'Neill PJ, Cotton BA, et al. Prevalence and risk factors for development of delirium in burn intensive care unit patients. J Burn Care Res 2010;31:706-15.

8. Zaal IJ, Devlin JW, Peelen LM, et al. A systematic review of risk factors for delirium in the ICU. Crit Care Med 2015;43:40-7.

9. Pandharipande PP, Girard TD, Jackson JC, et al. Long-term cognitive impairment after critical illness. N Engl J Med 2013;369:1306-16.

10. Guillamondegui OD, Richards JE, Ely EW, et al. Does hypoxia affect intensive care unit delirium or long-term cognitive impairment after multiple trauma without intracranial hemorrhage? J Trauma 2011;70:910-15.

11. Devlin JW, Fraser GL, Ely EW, et al. Pharmacological management of sedation and delirium in mechanically ventilated ICU patients: remaining evidence gaps and controversies. Semin Respir Crit Care Med 2013;34:201-15.

12. Mehta S, Cook D, Devlin JW, et al, SLEAP Investigators; Canadian Critical Care Trials Group. Prevalence, risk factors, and outcomes of delirium in mechanically ventilated adults. Crit Care Med 2015;43:557-66.

13. Lundström M, Edlund A, Karlsson S, et al. A multifactorial intervention program reduces the duration of delirium, length of hospitalization, and mortality in delirious patients. J Am Geriatr Soc 2005;53:622-8.

14. Thomason JW, Shintani A, Peterson JF, et al. Intensive care unit delirium is an independent predictor of longer hospital stay: a prospective analysis of 261 non-ventilated patients. Crit Care 2005;9:R375-81.

15. Mu JL, Lee A, Joynt GM. Pharmacologic agents for the prevention and treatment of delirium in patients undergoing cardiac surgery: systematic review and metaanalysis. Crit Care Med 2015;43:194-204.

16. Pauley E, Lishmanov A, Schumann S, et al. Delirium is a robust predictor of morbidity and mortality among critically ill patients treated in the cardiac intensive care unit. Am Heart $J$ 2015;170:79-86.e1.

17. Girard TD, Jackson JC, Pandharipande PP, et al. Delirium as a predictor of long-term cognitive impairment in survivors of critical illness. Crit Care Med 2010;38:1513-20.

18. Jackson JC, Gordon SM, Hart RP, et al. The association between delirium and cognitive decline: a review of the empirical literature. Neuropsychol Rev 2004;14:87-98.

19. Pun BT, Ely EW. The importance of diagnosing and managing ICU delirium. Chest 2007;132:624-36.

20. Hipp DM, Ely EW. Pharmacological and nonpharmacological management of delirium in critically ill patients. Neurotherapeutics 2012;9:158-75

21. Serafim RB, Bozza FA, Soares M, et al. Pharmacologic prevention and treatment of delirium in intensive care patients: a systematic review. J Crit Care 2015;30:799-807. 
22. Neerland BE, Hov KR, Bruun Wyller V, et al. The protocol of the Oslo Study of Clonidine in Elderly Patients with Delirium; LUCID: a randomised placebo-controlled trial. BMC Geriatr 2015;15:7.

23. Gagnon DJ, Riker RR, Glisic EK, et al. Transition from dexmedetomidine to enteral clonidine for ICU sedation: an observational pilot study. Pharmacotherapy 2015;35:251-9.

24. Trogrlic Z, van der Jagt M, Bakker J, et al. A systematic review of implementation strategies for assessment, prevention, and management of ICU delirium and their effect on clinical outcomes. Crit Care 2015;19:157.

25. Balas MC, Vasilevskis EE, Burke WJ, et al. Critical care nurses' role in implementing the "ABCDE bundle" into practice. Crit Care Nurse 2012;32:35-8, 40-7; quiz 48.

26. Weinhouse GL, Schwab RJ, Watson PL, et al. Bench-to-bedside review: delirium in ICU patients-importance of sleep deprivation. Crit Care 2009;13:234

27. King MS, Render ML, Ely EW, et al. Liberation and animation: strategies to minimize brain dysfunction in critically ill patients. Semin Respir Crit Care Med 2010;31:87-96.

28. Nakkeeran N, Zodpey SP. Qualitative research in applied situations: strategies to ensure rigor and validity. Indian J Public Health 2012;56:4-11.

29. Nakkeeran N. Knowledge, truth and social reality: an introductory note on qualitative research. Indian J Community Med 2010;35:379-81.

30. Kuper A, Reeves S, Levinson W. An introduction to reading and appraising qualitative research. BMJ 2008a;337:404-9.

31. Cunningham PJ, Felland LE, Ginsburg PB, et al. Qualitative methods: a crucial tool for understanding changes in health systems and health care delivery. Med Care Res Rev 2011;68:34-40.

32. Weiner BJ, Amick HR, Lund JL, et al. Use of qualitative methods in published health services and management research: a 10-year review. Med Care Res Rev 2011;68:3-33.

33. Carpenter C, Suto M. Qualitative research for occupational and physical therapists: a practical guide. Oxford: Black-Well Publishing, 2008.

34. Barbour RS. Making sense of focus groups. Med Educ 2005;39:742-50.

35. Keane S, Lincoln M, Smith T. Retention of allied health professionals in rural new South Wales: a thematic analysis of focus groups discussions. BMC Health Serv Res 2012;12:175.

36. Stickney CA, Ziniel SI, Brett MS, et al. Family participation during intensive care unit rounds: goals and expectations of parents and health care providers in a tertiary pediatric intensive care unit. $J$ Pediatr 2014;165:1245-51.

37. Nicholas DB, Hendson L, Reis MD. Connection versus disconnection: examining culturally competent care in the neonatal intensive care unit. Soc Work Health Care 2014;53:135-55.

38. Cachón-Pérez JM, Alvarez-López C, Palacios-Ceña D. [Non-pharmacological steps for the treatment of acute confusional syndrome in the intensive care unit]. Enferm Intensiva 2014;25:38-45.

39. Gearing RE. Bracketing in research: a typology. Qual Health Res 2004;14:1429-52

40. Tong A, Sainsbury P, Craig J. Consolidated criteria for reporting qualitative research (COREQ): a 32-item checklist for interviews and focus groups. Int J Qual Health Care 2007;19:349-57.

41. Teddlie C, Yu F. Mixed methods sampling a typology with examples. J Mixed Methods Res 2007;1:77.

42. Sasson C, Forman J, Krass D, et al. A qualitative study to identify barriers to local implementation of prehospital termination of resuscitation protocols. Circ Cardiovasc Qual Outcomes 2009;2:361-8.

43. Yevchak A, Steis M, Dile T, et al. Managing delirium in the acute care setting: a pilot focus group study. Int $J$ Older People Nurs 2012;7:152-62.

44. Giannini A, Garrouste-Orgeas M, Latour JM. What's new in ICU visiting policies: can we continue to keep the doors closed? Intensive Care Med 2014:40:730-3.

45. Willgerodt MA. Using focus groups to develop culturally relevant instruments. West J Nurs Res 2003;25:798-814.
46. Carpenter $\mathrm{C}$. Using qualitative focus groups to evaluate health programmes and service delivery. In Whalley Hammell K, Carpenter C, eds. Qualitative research in evidence-based rehabilitation. London: Churchill Livingstone, 2004:51-64.

47. Bloor M, Frankland J, Thomas M, et al. Focus groups in social research. London: Sage, 2001.

48. American Psychiatric Association. Diagnostic and Statistical Manual of Mental Disorders Fourth Edition. Text Revision. DSM-IV-TR Washington DC: American Psychiatric Association, 2001.

49. Braun V, Clarke V. Using thematic analysis in psychology. Qual Res Psychol 2006;3:77-101.

50. Saldaña J. The Coding Manual for Qualitative Researchers. Thousand Oaks, CA: Sage, 2014.

51. Casey D, Murphy K. Issues in using methodological triangulation in research. Nurse Res 2009;16:40-55.

52. Jung JH, Lim JH, Kim EJ, et al. The experience of delirium care and clinical feasibility of the CAM-ICU in a Korean ICU. Clin Nurs Res 2013;22:95-111.

53. Celis-Rodríguez E, Birchenall C, de la Cal MA, et al, Federación Panamericana e Ibérica de Sociedades de Medicina Crítica y Terapia Intensiva. Clinical practice guidelines for evidence-based management of sedoanalgesia in critically ill adult patients. Med Intensiva 2013;37:519-74.

54. Barr J, Fraser GL, Puntillo K, et al. Clinical practice guidelines for the management of pain, agitation, and delirium in adult patients in the intensive care unit. Crit Care Med 2013:41:263-306.

55. NICE. Delirium: diagnosis, prevention and management. The National Institute for Health and Clinical Excellence, 2010 (cited 16 September 2015). http://www.nice.org.uk/guidance/cg103/evidence/ cg103-delirium-full-guideline3

56. NICE. Delirium: evidence update April 2012. A summary of selected new evidence relevant to NICE clinical guideline 103 delirium: diagnosis, prevention and management 2010. The National Institute for Health and Clinical Excellence, 2012 (cited 16 September 2015). http://www.nice.org.uk/guidance/cg103/evidence/cg103-deliriumevidence-update2

57. Elliott SR. ICU delirium: a survey into nursing and medical staff knowledge of current practices and perceived barriers towards ICU delirium in the intensive care unit. Intensive Crit Care Nurs 2014;30:333-8

58. Fan $\mathrm{Y}, \mathrm{Guo} \mathrm{Y}, \mathrm{Li} \mathrm{Q}$, et al. A review: nursing of intensive care unit delirium. J Neurosci Nurs 2012;44:307-16; quiz E9-10.

59. Adams CL, Scruth EA, Andrade C, et al. Implementing clinical practice guidelines for screening and detection of delirium in a 21-hospital system in northern California: real challenges in performance improvement. Clin Nurse Spec 2015;29:29-37.

60. Merino P, Álvarez J, Cruz Martín M, et al; SYREC Study Investigators. Adverse events in Spanish intensive care units: the SYREC study. Int J Qual Health Care 2012;24:105-13.

61. Kamdar BB, Niessen T, Colantuoni $\mathrm{E}$, et al. Delirium transitions in the medical ICU: exploring the role of sleep quality and other factors. Crit Care Med 2015;43:135-41.

62. Caruso P, Guardian L, Tiengo T, et al. ICU architectural design affects the delirium prevalence: a comparison between single-bed and multibed rooms*. Crit Care Med 2014;42:2204-10.

63. Mistraletti G, Carloni E, Cigada M, et al. Sleep and delirium in the intensive care unit. Minerva Anestesiol 2008;74:329-33.

64. Rock LF. Sedation and its association with posttraumatic stress disorder after intensive care. Crit Care Nurse 2014;34:30-7; quiz 39.

65. Bruno JJ, Warren ML. Intensive care unit delirium. Crit Care Nurs Clin North Am 2010;22:161-78.

66. Reade MC, Finfer S. Sedation and delirium in the intensive care unit. N Engl J Med 2014;370:444-54.

67. Linton J, Farrell MJ. Nurses' perceptions of leadership in an adult intensive care unit: a phenomenology study. Intensive Crit Care Nurs 2009;25:64-71.

68. Ista E, Trogrlic Z, Bakker J, et al. Improvement of care for ICU patients with delirium by early screening and treatment: study protocol of iDECePTIvE study. Implement Sci 2014:9:143.

69. Koch T, Kralik D. Participatory action research in health care. Oxford: Blackell Publishing, 2006. 